\title{
A intervenção "Ensino do cuidado com os pés" para pessoas com diabetes: ensaio clínico randomizado
}

\author{
Intervention "Teaching foot care" for people with diabetes: a randomized clinical trial
}

\section{iD Lidiane Aparecida Monteiro ${ }^{1}$ \\ Denise Hollanda Iunes ${ }^{2}$ \\ (iD) Ana Emília Pace ${ }^{3}$ \\ (iD) Denismar Alves Nogueira ${ }^{4}$ \\ (iD) João Batista Moreira ${ }^{5}$ \\ Érika de Cássia Lopes Chaves ${ }^{6}$}

Parecer de aprovação pelo comitê de ética CAAE: 20376013.2.0000.5142

\section{Autor correspondente:}

Érika de Cássia Lopes Chaves

\section{Endereço Científico:}

Universidade Federal de Alfenas, Rua Gabriel Monteiro da Silva, 700 - Centro, Alfenas - MG, 37130001

${ }^{1}$ Doutorado pela Universidade de São Paulo. lidianemonteiro22@hotmail.com

${ }^{2}$ Pós-doutorado pela Universidade de São Paulo, Professora na Universidade Federal de Alfenas. deniseiunes@unifal-mg.edu.br

${ }^{3}$ Pós-doutorado pela Universidade de São Paulo, Professora na Universidade Federal de São Paulo. aepace@eerp.usp.br

${ }^{4}$ Doutorado pela Universidade Federal de Lavras, Professor de estatística na Universidade Federal de Alfenas.denisnog@gmail.com

${ }^{5}$ Mestre pela Universidade Federal de Alfenas. joaobatistamoreira@yahoo.com.br

${ }^{6}$ Pós-doutorado pela Universidade de São Paulo, Professora na Universidade Federal de Alfenas.

"bolsista do Programa de Educação Tutorial - PET ENFERMAGEM - Docente da Escola de Enfermagem da UNIFAL-MG"

echaves@unifal-mg.edu.br

\section{Resumo}

Introdução: pessoas com diabetes possuem risco aumentado para o desenvolvimento de morbidades nos membros inferiores, o que está relacionado a amputações.

Objetivo: analisar o efeito da intervenção "Ensino do Cuidado com os Pés", realizada por meio de visitas domiciliares, na integridade tissular, no conhecimento sobre as atividades de autocuidado e na intenção de cuidar dos pés.

Método: ensaio clínico paralelo, de mascaramento único e randomizado com taxa de alocação de 1:1, desenvolvido com pessoas com Diabetes tipo 2. O desfecho primário está relacionado a avaliação da integridade tissular e o secundário, ao conhecimento sobre as atividades de autocuidado e à intenção em realizar cuidados com os pés.

Resultados: Participaram do estudo 72 pessoas, divididas em grupo controle e tratado. $\mathrm{O}$ grupo controle apresentou diferença significativa $(\mathrm{p}=<0,05)$ nos indicadores de pele e anexo, circulação, sensibilidade, conhecimento e intenção de cuidar dos pés.

Conclusão: "Ensino do Cuidado com os Pés" é uma estratégia que pode influenciar os indicadores de comprometimento dos pés. $\mathrm{n}^{\circ}$ 20376013.2.0000.5142

Descritores: Pé diabético. Diabetes mellitus. Educação em saúde. Enfermagem.

\section{Abstract}

Introduction: people with diabetes are at increased risk for developing lower limbs morbidities, which are related to amputations.

Objective: analyzing the effect of the intervention "Teaching Foot Care", carried out through home visits, on tissue integrity, on knowledge about selfcare activities and on the intention of foot care.

Method: parallel, single-blind, randomized clinical testing with an allocation rate of 1:1, developed with people with type 2 diabetes. The primary outcome is related to the evaluation of tissue integrity and the secondary is related to knowledge about self-care activities and the intention to perform foot care.

Results: 72 people participated in the study, divided into control and treated groups. Control group showed a significant difference $(\mathrm{p}=<0.05)$ in the indicators of skin and its attachments, circulation, sensitivity, knowledge and intention to take care of the feet.

Conclusion: "Teaching Foot Care" is a strategy that can influence indicators of foot impairment. $\mathrm{N}^{\circ}$. 20376013.2.0000.5142

Keywords: Diabetic foot. Diabetes mellitus. Health education. Nursing.

\section{Cite como}

Vancouver

Monteiro, LA, Iunes, DH, Pace, AE, Nogueira, DA, Moreira, JB, Chaves, ÉCL. A intervenção "Ensino do cuidado com os pés" para pessoas com diabetes: ensaio clínico randomizado. Conscientiae Saúde 2021;20:1-18, e19889. https://doi.org/10.5585/20.2021.19889. 


\section{Introdução}

As morbidades que afetam as extremidades dos membros inferiores em pessoas com Diabetes Mellitus (DM) são destacadas por representarem um problema frequente, complexo e dispendioso, não somente para a pessoa e familiares, como também para a saúde pública ${ }^{1}$. A neuropatia diabética associada a outros agravos, como as doenças vasculares periféricas, determinam essa importante condição clínica, que denomina-se "pé diabético", caracterizada pela presença de infecção, ulceração e/ou destruição de tecidos profundos ${ }^{2}$.

A incidência e a prevalência das úlceras em pés (UPDs) das pessoas com DM são mais elevadas do que em outras situações clínicas, 2 a $4 \%$ e 4 a 10\%, respectivamente ${ }^{1}$. Em cinco anos, o risco de morte para uma pessoa com DM e úlcera plantar é 2,5 vezes maior, quando comparado a outra com o mesmo diagnóstico sem complicações nos pés ${ }^{3}$.

Em Piauí, Teresina, um estudo epidemiológico transversal, analítico, com o objetivo de analisar a prevalência de lesão por pressão, úlcera diabética e vasculogênica, realizado nos serviços de atenção básica com 339 idosos demonstrou que 25,9\% da amostra do estudo apresentavam úlceras diabéticas, sendo $80 \%$ em região plantar ${ }^{4}$.

Devido a graves repercussões na saúde e na sociedade, a úlcera plantar deve ser prevenida e, neste contexto de atenção à pessoa com DM, estratégias educacionais com participação ativa é uma alternativa viável, uma vez que permite estimular o comportamento saudável necessário para o cuidado com a doença ${ }^{5}$, em especial com os pés.

Entre as estratégias educativas individualizadas, direcionadas a pessoa com DM, destaca-se a realizada no domicílio, que facilita identificar as particularidades do cuidado. $\mathrm{O}$ enfermeiro que atua na atenção domiciliar deve ser sensível às demandas e necessidades das pessoas assistidas e, assim, utilizar o ambiente de aprendizagem para avaliar, projetar, desenvolver e implementar um plano de ensino individualizado para cada situação, na busca pela construção de conhecimentos e habilidades necessários com a doença ${ }^{6}$.

Na região Sudeste, em São Paulo, Ribeirão Preto foi desenvolvido um ensaio clínico randomizado com 164 pessoas com DM e com o objetivo de avaliar a contribuição do apoio social familiar por meio de intervenções educativas. Os resultados mostraram que o grupo intervenção apresentou diferenças na pressão arterial sistêmica e hemoglobina glicada quando comparado ao grupo controle ${ }^{7}$.

Para desenvolver a educação em saúde, recomenda-se ainda que o enfermeiro utilize linguagens e terminologias de enfermagem padronizadas internacionalmente, tais como a North American Nursing Diagnosis Association (NANDA) ${ }^{8}$, Nursing Outcomes Classification 
$(\mathrm{NOC})^{9}$ e Nursing Interventions Classification (NIC) ${ }^{10}$, para descrever e definir os diagnósticos, resultados e intervenções de enfermagem, respectivamente ${ }^{11}$.

O DM mau controlado por um logo período de tempo contribui para o desequilíbrio metabólico e nutricional, bem como para o desenvolvimento de morbidades importantes, acarretando comprometimentos micro e macro vasculares ${ }^{12}$, os quais se destacam no desenvolvimento das úlceras em pés, a neuropatia diabética e doença arterial, respectivamente.

Este estudo visa analisar o efeito da intervenção de enfermagem "Ensino do Cuidado com os Pés" na integridade tissular, no conhecimento sobre as atividades de autocuidado e na intenção de cuidar dos pés de pessoas com DM tipo 2.

\section{Método}

Tipo de estudo

Trata-se de um ensaio clínico paralelo, de mascaramento único e randomizado com taxa de alocação de 1:1. As avaliações foram feita por um pesquisador independente e cego quanto às intervenções. $\mathrm{O}$ estudo foi realizado em unidade cujo modelo de atenção está estruturado na Estratégia de Saúde da Família (ESF) no estado de Minas Gerais, no período de janeiro de 2014 a Janeiro de 2015.

\section{População}

A população foi constituída por 290 pessoas com DM tipo 2, cadastradas no Sistema de Informação de Atenção Básica (SIAB), identificadas por meio da busca no cadastro ativo da unidade.

\section{Critérios de seleção}

Como critérios de elegibilidade foram consideradas pessoas com DM tipo 2 diagnosticadas há, no mínimo, cinco anos e com idade acima de dezoito anos. Foram excluídas pessoas com DM tipo 2 que apresentavam lesões nos pés, amputações nos membros inferiores e déficit na capacidade cognitiva identificado pelo Mini-Exame do Estado Mental (MEEM) ${ }^{13}$.

\section{Definição da amostra}

Para o cálculo amostral ${ }^{14}$, foi desenvolvido um teste piloto com dez participantes. Considerando um nível de significância de $5 \%$ e uma probabilidade de $20 \%$ de se cometer o 
erro tipo II, o cálculo foi embasado nos escores do resultado de enfermagem "Integridade Tissular: pele e mucosas ${ }^{15}$ ". O resultado obtido para o tamanho amostral foi de no mínimo 70 participantes.

$$
n=\frac{\left[z_{\left(\frac{\alpha}{2}\right)} \sqrt{2 \pi(1-\pi)}+z_{(\beta)} \sqrt{\pi_{1}\left(1-\pi_{1}\right)}+\pi_{2}\left(1-\pi_{2}\right)\right]^{2}}{\delta^{2}}
$$

$\alpha=$ taxa de erro tipo I $-5 \%$

$\beta=$ taxa de erro tipo II $-20 \%$

$\pi 1=$ taxa de eventos esperados no grupo de controle $(0)$

$\pi 2=$ taxa de eventos esperados no grupo tratado $(20 \%)$

$\pi=(\pi 1+\pi 2) / 2$

$\square=\pi 1-\pi 2$

$\mathrm{z}(\alpha / 2)=$ constante da distribuição normal padrão dependendo do valor de $\alpha$

$\mathrm{z}(\beta)=$ constante da distribuição normal padrão dependendo do valor de $\beta$

A randomização ocorreu por meio de dois braços distintos, denominados Grupo Tratado (GT) e Grupo Controle (GC). A distribuição dos participantes entre os grupos foi realizada por meio do programa Random.org.

\section{Coleta de dados}

Para atender aos objetivos do presente estudo, foi determinado o diagnóstico de enfermagem "risco de integridade da pele prejudicada (00047)" dos pés, tendo em vista que as pessoas com DM podem apresentar fatores de risco tais como a circulação prejudicada, o estado nutricional desequilibrado, o estado metabólico prejudicado, as deformações ósseas e as sensações prejudicadas ${ }^{8}$.

Os resultados esperados estão embasados nos indicadores propostos pela Nursing Outcomes Classification - NOC ${ }^{9}$ para o resultado "Integridade Tissular: pele e mucosas" (1101), além de outros identificados na literatura ${ }^{16}$.

Desta forma, nos dados sociodemográficos foram descritos o nome do participante, data de nascimento, unidade de origem, hábitos de vida e aspectos clínicos. O rastreamento do comprometimento cognitivo foi realizado por meio do MEEM ${ }^{13}$. 
A medida do desfecho primário, trata-se da análise do resultado da intervenção "Ensino do Cuidado com os Pés" na redução dos riscos de complicações plantares em pessoas com DM tipo 2. Foi definido o risco de complicações, tendo em vista que essas pessoas podem apresentar integridade tissular preservada, mas possuem o risco de desenvolvimento de complicações determinado pelas características da doença, bem como pelo seu mau controle. Assim, foi avaliado antes e após as intervenções educativas por meio da "Escala de Avaliação da "Integridade Tissular: Pele e Mucosas" dos Pés de Pacientes com Diabetes Melittus tipo II". As respostas variam entre cinco pontos na escala tipo Likert em que "1" representa extremamente comprometido; "2" substancialmente comprometido; “3" moderadamente comprometido; "4" levemente comprometido, e; "5" não comprometido"

Para avaliar o desfecho referente à pele e mucosas, incluiu-se a observação dos MMII em busca de lesão tecidual, crescimento de pelos na pele, elasticidade/turgor, hidratação, transpiração, textura, espessura, cor da pele com MMII abaixados, cor da pela após 10 segundos de elevação dos MMII a $30 \mathrm{~cm}$ de altura, pigmentação, integridade da pele/descamação cutânea ${ }^{9,15}$.

A circulação sanguínea foi avaliada por meio dos seguintes indicadores: digitopressão do hálux para identificar a perfusão tissular; contagem da frequência dos pulsos pedioso e tibial posterior compressão dos MMII para identificação de edema e visualização dos MMII em busca de varizes ${ }^{8,9,13,15,16,17}$.

A sensibilidade tátil pressórica foi avaliada em quatro áreas plantares específicas: hálux, $1^{\mathrm{a}}, 3^{\mathrm{a}}$ e $5^{\mathrm{a}}$ cabeças de metatarsos, por meio do Monofilamento de Semmes-Weinstein 5.07, de $10 \mathrm{~g}^{17}$, e avaliado em presença ou ausência de sensibilidade. Em complemento, foi utilizado o Escore de Sintomas Neuropáticos $(\mathrm{ESN})^{9,15,17}$, que os avalia numa escala 3-4 que implica em sintomas leves; 5-6 sintomas moderados e 7-9 sintomas graves.

Para mensurar a temperatura, foi utilizado o termômetro de superfície infravermelho em dezoito pontos dos pés ${ }^{9,15}$, e considerado temperatura entre $36^{\circ} \mathrm{C}$ a $38^{\circ} \mathrm{C}$ dentro dos parâmetros normais e, de $35^{\circ} \mathrm{C}$ a $37^{\circ} \mathrm{C}$ para idosos ${ }^{17}$.

As áreas de pressão plantar foram identificadas por meio do baropodômetro formado por uma plataforma modular contendo sensores eletrônicos de platina, o qual permite avaliar a distribuição do apoio estático por meio de imagens, durante a posição ortostática ${ }^{15}$. A pressão plantar dinâmica ou estática são consideradas normais se máxima abaixo de $89,22 \mathrm{Kg} / \mathrm{cm}^{2} \mathrm{em}$ ambos os pés ${ }^{15}$. 
O desfecho secundário está relacionado ao conhecimento sobre as atividades de autocuidado e à intenção das pessoas com DM em realizar tais cuidados com os pés e, foram avaliados também, antes e após as intervenções educativas.

O Questionário de Avaliação do Conhecimento sobre o Autocuidado com os Pés de Pessoas com Diabetes Mellitus tipo 2 foi elaborado, especificamente para este estudo, com o objetivo de avaliar o conhecimento dos participantes do estudo sobre as atividades de autocuidado com os pés. Destaca-se que tais atividades recomendadas pelo MS foram submetidas a um processo de refinamento por um comitê de juízes peritos no assunto, os quais propuseram adequações para se obter a versão ideal do questionário. O questionário é composto por 16 perguntas, com respostas dicotômicas (Sim-0 e Não-1). Foi considerado baixo conhecimento (até quatro respostas "Sim"), moderado conhecimento (quatro a oito respostas "Sim"), alto conhecimento (oito a 12 respostas "Sim") ou muito alto conhecimento (12 a 16 respostas "Sim").

O Questionário do comportamento planejado - cuidado co os pés (QCP-CP) trata-se de um instrumento de origem portuguesa com o objetivo de identificar a intenção das pessoas com DM em realizar as atividades de autocuidado com os pés. O instrumento é composto por seis domínios: "Intenções", “Atitudes", "Normas subjetivas", "Controle Comportamental Percebido", "Planejamento da Ação" e "Planejamento do Coping". As respostas são dadas em uma escala tipo Likert, de cinco pontos. $\mathrm{O}$ instrumento não tem ponto de corte definido, quanto maior a pontuação, melhor é a intenção de cuidar dos pés. Vale lembrar que os domínios "Atitudes" e "Normas Subjetivas" possuem escores invertidos. O QCP-CP foi adaptado para a cultura brasileira e validado ${ }^{18}$.

O desenvolvimento do presente estudo foi constituído por duas sessões de avaliação e oito sessões de intervenção. As avaliações foram realizadas na sessão zero, antes de iniciar a intervenção educativa, e após a última intervenção, denominada de avaliação final. Para a coleta de dados, os voluntários foram recebidos em uma sala de apoio, situada na área de abrangência da ESF, a qual continha ar condicionado, visando garantir o controle da temperatura do ambiente.

A intervenção "Ensino do Cuidado com os Pés", direcionada ao GT, foi elaborada fundamentada nas atividades de autocuidado propostas pela Nursing Interventions Classification (NIC), pelo MS e fontes oriundas da literatura ${ }^{16}$. A intervenção foi realizada por um período de quatro meses a partir das seguintes estratégias: orientação escrita - folder ilustrativo; visita domiciliária - quinzenalmente; orientação verbal e feedback e observação da adoção/execução das atividades. 
No primeiro dia agendado para visita domiciliar, o voluntário recebia a orientação escrita e logo era realizado a orientação verbal das atividades de autocuidado com os pés. Assim, era incentivado o feedback do voluntário sobre o seu conhecimento e a sua prática em relação aos cuidados com os pés. Após esse momento, era observado os pés. Nas visitas domiciliares subsequentes, a intervencionista primeiramente observava os pés para verificar a adoção das atividades orientadas, e reforçava os cuidados descritos no folder de forma singular, a depender da necessidade e conhecimento de cada voluntário.

Os voluntários alocados no GC também receberam visitas domiciliares quinzenais, pelo período de quatro meses, contudo, não era ofertado nenhum tipo de intervenção específica. A intervencionista perguntava sobre os cuidados com diabetes de forma geral e deixava a pessoa explanar sobre seus medos, anseios e dúvidas. Assim, a visita era pautada basicamente pela escuta qualificada.

\section{Análise e tratamento dos dados}

Os dados coletados foram tabulados no Excel 2007 e validados, sendo posteriormente importados pelos programas R, versão 3.1.1, e SPSS versão 21,0. Foi realizada a estatística descritiva para os dados da caracterização sociodemográficas. Para o estudo da relação entre as variáveis qualitativas, foi utilizado o teste Qui-Quadrado ou o Teste Exato de Fisher, de acordo com a distribuição condicional.

O teste de normalidade utilizado foi Shapiro-Wilk a 5\%. Na comparação das variáveis contínuas dos GT e GC e de cada grupo ao longo das avaliações, foi utilizado Anova e para as variáveis discretas, o Teste de Wilcoxon. A análise do desfecho primário foram utilizados Teste de Wilcoxon para as variáveis pele e anexos, circulação e sensibilidade e Anova para temperatura e pressão plantar. Especificamente para o desfecho secundário, utilizou-se Anova para o conhecimento e intenção de cuidar. A análise interina e por intenção de tratar foram descartadas. Destaca-se que os testes foram aplicados considerando-se um nível de significância de $5 \%$.

Para identificar o poder estatístico do estudo, foi realizada a análise dos resultados, por meio do programa Piface Application Selector. Como referência, utiliza-se um poder estatístico acima de $80 \%$. 


\section{Aspectos éticos}

O projeto foi aprovado pelo Comitê de Ética em Pesquisa da Universidade Federal de Alfenas e registrado na Plataforma de Registro Brasileiro de Ensaios Clínicos. Os participantes do estudo assinaram o termo de consentimento livre e esclarecido em duas vias.

\section{Resultados}

Para o rastreamento da amostra foram analisados os cadastros das pessoas com DM atendidas pela ESF. Assim, foram identificadas nove pessoas diagnosticadas com DM tipo 1 e 119 com menos de cinco anos de diagnóstico de DM tipo 2, no entanto, 162 atendiam aos critérios de inclusão e foram consideradas para o primeiro contato por meio de visita domiciliar.

Dentre estas, 99 receberam a visita domiciliar, tendo em vista que 63 não foram localizadas por motivo de mudança, horário de trabalho, atividades na zona rural ou em outra cidade, viagem, hospitalização ou falecimento; cinco recusaram participar do estudo e doze apresentavam critérios de exclusão.

No total, 82 pessoas corresponderam aos critérios de elegibilidade, das quais, 10 foram alocadas para o grupo pré-teste e 72, para o estudo propriamente dito. A randomização ocorreu por meio de dois braços distintos $\mathrm{GT}=36$ pessoas e $\mathrm{GC}=36$ pessoas, o que está em consonância com os resultados do cálculo amostral. Permaneceram até o final do estudo, 67 pessoas, ou seja, 35 no GT e 32 no GC devido a perdas no seguimento, respectivamente, de uma pessoa no GT e quatro no GC (Figura 1). 
Figura 1 - Fluxograma de rastreamento da amostra conforme Consort
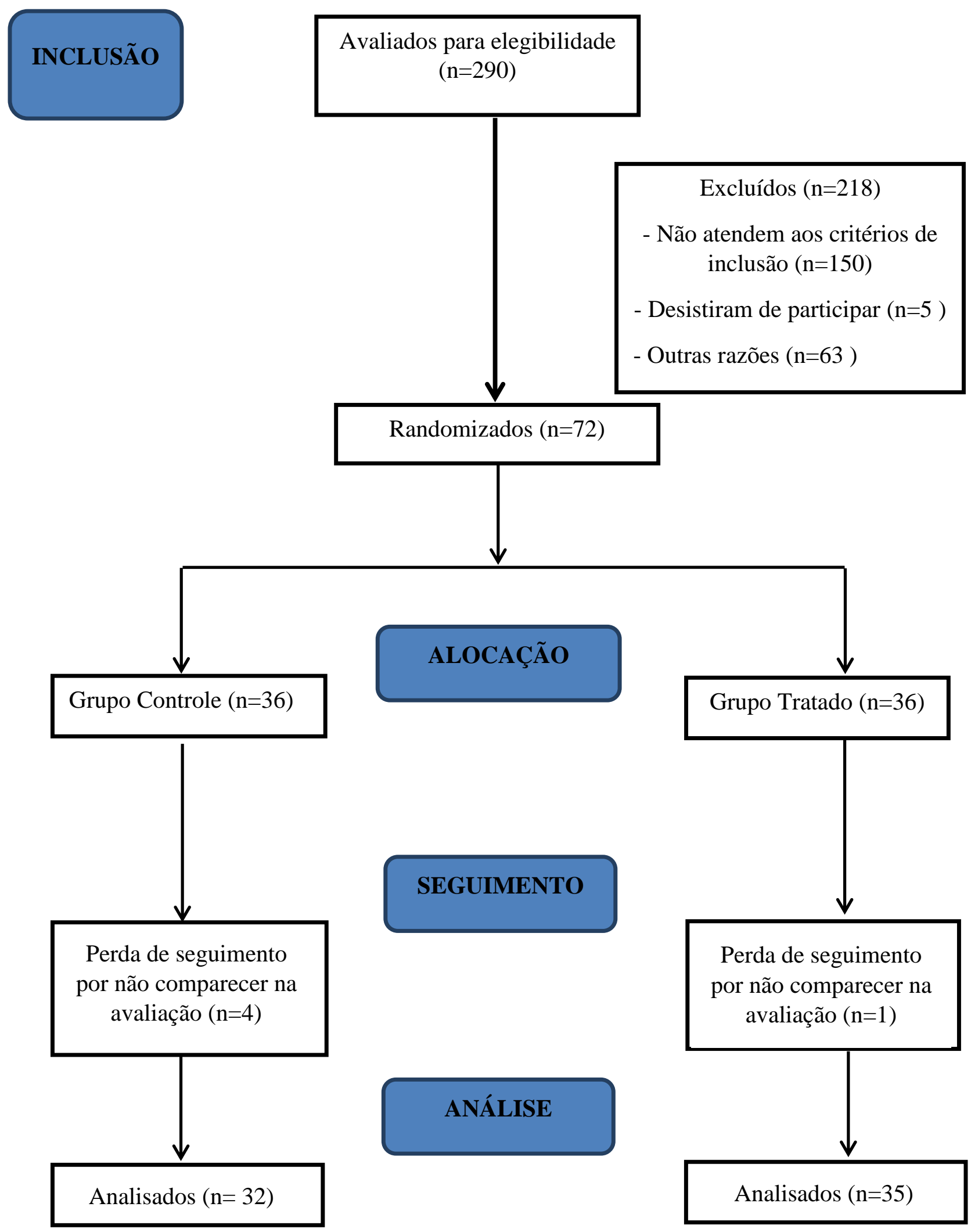

Fonte: Autores. 
O perfil da amostra final do estudo foi representado por variáveis sóciodemográficas e aspectos clínicos tais como, tipo de tratamento, associação de hipertensão ou outras doenças. Os grupos apresentaram-se homogêneos em relação ao sexo, escolaridade e renda. Referente à avaliação dos pés e ao recebimento de orientações de autocuidado, observa-se que a maioria dos voluntários não receberam orientações sobre cuidados com os pés e nem tiveram seus pés avaliados (Tabela 1).

Tabela 1 - Distribuição por frequência absoluta dos grupos de acordo com gênero, nível de escolaridade, renda familiar e dos aspectos relacionados à avaliação dos pés e orientação dos cuidados com os pés. Minas Gerais, 2014. ( $\mathrm{n}=67)$

\begin{tabular}{|c|c|c|c|c|}
\hline \multicolumn{2}{|c|}{ CARACTERÍSTICAS } & $\begin{array}{c}\text { Grupo Tratado } \\
\qquad(\mathbf{n}=\mathbf{3 5})\end{array}$ & $\begin{array}{c}\text { Grupo Controle } \\
\qquad(\mathrm{n}=\mathbf{3 2})\end{array}$ & $\begin{array}{c}\mathbf{p} \\
\text { Valor }\end{array}$ \\
\hline \multirow{2}{*}{ Sexo } & Feminino & $24(68,57 \%)$ & $19(59,37 \%)$ & \multirow{2}{*}{0,43} \\
\hline & Masculino & $11(31,43 \%)$ & $13(40,63 \%)$ & \\
\hline \multirow{6}{*}{ Escolaridade } & Nunca estudou & $12(34,30 \%)$ & $12(37,50 \%)$ & \multirow{6}{*}{0,72} \\
\hline & Fundamental incompleto & $14(40,00 \%)$ & $16(50,00 \%)$ & \\
\hline & Fundamental completo & & & \\
\hline & Ensino médio incompleto & $7(20,00 \%)$ & $3(9,37 \%)$ & \\
\hline & Ensino médio completo & $1(2,85 \%)$ & $1(3,13 \%)$ & \\
\hline & Ensino superior incompleto & $1(2,85 \%)$ & 0 & \\
\hline \multirow{5}{*}{ Renda em salários } & $1^{*}$ & $10(28,57 \%)$ & $10(31,25 \%)$ & \multirow{5}{*}{0,47} \\
\hline & 2 & $15(42,85 \%)$ & $13(40,62 \%)$ & \\
\hline & 3 & $7(20,00 \%)$ & $8(25,00 \%)$ & \\
\hline & 4 & $3(8,58 \%)$ & 0 & \\
\hline & 5 & 0 & $1(3,13 \%)$ & \\
\hline \multirow{2}{*}{$\begin{array}{l}\text { Recebeu avaliação dos } \\
\text { pés }\end{array}$} & Sim & $3(8,57 \%)$ & $3(50 \%)$ & \multirow{2}{*}{1,00} \\
\hline & Não & $32(91,43 \%)$ & $29(47,5 \%)$ & \\
\hline \multirow{2}{*}{$\begin{array}{l}\text { Recebeu orientação de } \\
\text { cuidados com os pés }\end{array}$} & Sim & $4(11,42 \%)$ & $4(12,50 \%)$ & \multirow[b]{2}{*}{1,00} \\
\hline & Não & $31(88,58 \%)$ & $28(87,50 \%)$ & \\
\hline
\end{tabular}

*Valor do salário mínimo $=\overline{724,00}$ reais.

Fonte: Autores.

Os voluntários faziam tratamento com antidiabéticos orais $(\mathrm{GT}=71,42 \%$ e $\mathrm{GC}=$ 71,87\%, $\mathrm{p}=0,97)$, apresentavam hipertensão arterial autorreferida (GT $=85,71 \%$ e $\mathrm{GC}=$ $90,62 \%, \mathrm{p}=0,71)$ e possuíam outras doenças associadas ( $\mathrm{GT}=57,14 \%$ e $\mathrm{GC}=43,75 \%, \mathrm{p}=27)$, tais como cardiopatias, distúrbios musculoesqueléticos e neuropsíquicos.

No desfecho primário, avaliado pela Escala de Avaliação da "Integridade Tissular: Pele e Mucosas", o GT apresentou diferença significativa, quando comparado a primeira e segunda avaliação $(\mathrm{p}<0,05)$ (poder estatístico > 80\%). (Tabela 2). 
Tabela 2 - Distribuição das médias do risco global de integridade tissular dos pés (Pele e anexo, Circulação, Sensibilidade, Temperatura tissular, Pressão plantar). Minas Gerais, 2014. $(\mathrm{n}=67)$

\begin{tabular}{ccc}
\hline Risco de Integridade tissular dos pés & \\
\hline Grupo Tratado & $\mathbf{1}^{\mathrm{a}}$ Avaliação & $\mathbf{2}^{\mathrm{a}}$ Avaliação \\
Grupo Controle & $79,20^{\mathrm{bA}}$ & $\mathbf{8 5 , 7 4}$ \\
\hline
\end{tabular}

Fonte: Autores.

Médias seguidas de mesma letra minúscula na mesma linha são estatisticamente iguais pelo teste F da Anova a 5\%. Médias seguidas de mesma letra maiúscula na mesma coluna são estatisticamente iguais pelo teste $\mathrm{F}$ a 5\%. (Linha: comparações no tempo e Coluna: comparações no grupo). ${ }^{1}$ Poder estatístico $>80 \%$.

Em relação a "Escala de Avaliação da "Integridade Tissular: Pele e Mucosas" dos Pés de Pacientes com Diabetes Mellitus tipo II”, observa-se que nos domínios Pele e Anexo e Circulação houve diferença do GT quando comparado a $1^{\mathrm{a}}$ com a $2^{\mathrm{a}}$ avaliação.

No domínio Sensibilidade houve diferença na $2^{\mathrm{a}}$ avaliação quando comparado ao Grupo controle, especificamente no ESN. Estes três domínios apresentaram um poder estatístico > $80 \%$.

Nos domínios Temperatura Tissular e Pressão Plantar não houveram diferenças e o poder estatístico foi $<70 \%$ (Tabela 3 ).

Tabela 3 - Distribuição das médias por domínios da "Escala de Avaliação da "Integridade Tissular: Pele e Mucosas" dos Pés de Pacientes com Diabetes Mellitus tipo II". Minas Gerais, 2014. $(\mathrm{n}=67)$

\begin{tabular}{|c|c|c|c|}
\hline Domínios & Grupos & $1^{a}$ Avaliação & $2^{a}$ Avaliação \\
\hline \multirow{2}{*}{ Pele e anexo ${ }^{1}$} & Tratado & $47,51^{\mathrm{aA}}$ & $51,22^{\mathrm{bA}}$ \\
\hline & Controle & $47,34^{\mathrm{aA}}$ & $48,43^{\mathrm{aB}}$ \\
\hline \multirow{2}{*}{ Circulaçãão $^{1}$} & Tratado & $20,34^{\mathrm{aA}}$ & $\mathbf{2 2 , 3 1 ^ { \mathrm { bA } }}$ \\
\hline & Controle & $20,65^{\mathrm{aA}}$ & $21,50^{\mathrm{aB}}$ \\
\hline \multirow{2}{*}{ Sensibilidade $^{1}$} & Tratado & $7,05^{\mathrm{aA}}$ & $7,80^{\mathrm{aA}}$ \\
\hline & Controle & $7,71^{\mathrm{aA}}$ & $7,15^{\mathrm{aB}}$ \\
\hline \multirow{2}{*}{ Temperatura tissular ${ }^{3}$} & Tratado & $1,08^{\mathrm{aA}}$ & $1,00^{\mathrm{aA}}$ \\
\hline & Controle & $1,04^{\mathrm{aA}}$ & $1,00^{\mathrm{aA}}$ \\
\hline \multirow{2}{*}{ Pressão plantar ${ }^{1}$} & Tratado & $3,12^{\mathrm{aA}}$ & $3,21^{\mathrm{aA}}$ \\
\hline & Controle & $3,30^{\mathrm{aA}}$ & $3,17^{\mathrm{aA}}$ \\
\hline
\end{tabular}

Fonte: Autores.

Médias seguidas de mesma letra minúscula na mesma linha são estatisticamente iguais pelo teste F da Anova a 5\%. Médias seguidas de mesma letra maiúscula na mesma coluna e mesmo domínio são estatisticamente iguais pelo teste $\mathrm{F}$ a 5\%. (Linha: comparações no tempo e Coluna: comparações no grupo). ${ }^{1}$ Poder estatístico $>80 \%$ e ${ }^{3}$ Poder estatístico $<70 \%$. 
Em relação à medida de desfecho secundário, avaliada pelo conhecimento e intenção de cuidar, houve diferença no conhecimento do GT na $1^{\mathrm{a}}$ avaliação em relação à $2^{\mathrm{a}}$ e em relação ao GC (poder estatístico > 80\%) (Tabela 4).

Tabela 4 - Distribuição das médias do resultado global do conhecimento sobre as atividades de autocuidado com os pés. Minas Gerais, 2014. $(\mathrm{n}=67)$

\begin{tabular}{cccc}
\hline Conhecimento das Atividades de Autocuidado com os Pés & $1^{\mathrm{a}}$ Avaliação & $2^{\mathrm{a}}$ Avaliação \\
\hline Grupo Tratado & $8,17^{\mathrm{Aa}}$ & $\mathbf{1 , 9 4}^{\mathrm{bA}}$ \\
Grupo Controle & $9,09^{\mathrm{aA}}$ & $7,53^{\mathrm{aB}}$ \\
\hline
\end{tabular}

Fonte: Autores.

Médias seguidas de mesma letra minúscula na mesma linha são estatisticamente iguais pelo Teste F da Anova a 5\%. Médias seguidas de mesma letra maiúscula na mesma coluna são estatisticamente iguais pelo teste $\mathrm{F}$ a 5\%. (Linha: comparações no tempo e Coluna: comparações no grupo). ${ }^{1}$ Poder estatístico $>80 \%$

$\mathrm{Na}$ avaliação da intenção de cuidar, observou-se que a média global obtida pelos voluntários do GT foi superior na segunda avaliação em relação ao GC (poder estatístico > $80 \%$ ) (Tabela 5).

Ressalta-se que a média de todas as variáveis do presente estudo aponta um poder estatístico de $98 \%$.

Tabela 5 - Distribuição das médias do resultado global da intenção de cuidar dos pés. Minas Gerais, 2014. $(n=67)$

\begin{tabular}{c|c|c}
\hline Intenção de Cuidar dos Pés $^{1}$ & 1 $^{\text {a }}$ Avaliação & 2 $^{\text {a }}$ Avaliação $^{\text {a }}$ \\
\hline Grupo Tratado & $94,34^{\mathrm{bA}}$ & $\mathbf{1 0 1 , 3 4}^{\mathrm{A}}$ \\
Grupo Controle & $94,96^{\mathrm{Ab}}$ & $94,18^{\mathrm{aB}}$
\end{tabular}

Fonte: Autores

Médias seguidas de mesma letra minúscula na mesma linha são estatisticamente iguais pelo Teste F da Anova a 5\%. Médias seguidas de mesma letra maiúscula na mesma coluna são estatisticamente iguais pelo teste F a 5\%. (Linha: comparações no tempo e Coluna: comparações no grupo). ${ }^{1}$ Poder estatístico > 80\%

\section{Discussão}

Ao avaliar os efeitos das intervenção "Ensino do Cuidado com os Pés" para manter a sua integridade, foi observado que os voluntários que receberam as intervenções apresentaram 
resultados significativos em indicadores de comprometimento dos pés, tais como pele e anexos, circulação e sensibilidade e, ainda, no conhecimento e intenção de cuidar dos pés (Valor $\mathrm{p}<0,05)$.

A melhora do GT nos escores de preservação da pele e anexos pode estar relacionada às orientações das atividades para a prevenção do risco de integridade tissular dos pés, tais como manter os pés limpos, hidratar os pés, secar entre os dedos, observar os pés diariamente, não cortar calos nem cutículas, procurar a equipe de profissionais da saúde quando apresentar alterações visíveis nos pés, não utilizar substâncias químicas, quentes ou frias nos pés, solicitar a ajuda da família nos cuidados com os pés, dentre outras ${ }^{17}$.

Semelhante aos resultados deste estudo, na Malásia um estudo quase experimental, realizado entre idosos diagnosticados com DM, identificou que após as intervenções educativas, as condições dos pés melhoraram, principalmente a higiene global dos pés ( $\mathrm{p}=$ $0,03)$ e a anidrose $(\mathrm{p}=0,02)^{19}$.

Resultados positivos também foram identificados na circulação sanguínea, especificamente nos indicadores de perfusão tissular, pulsos e edema, do GT após a implementação da intervenção educativa, o que pode estar relacionado com as orientações sobre a importância da manutenção de uma atividade física, alimentação saudável e a diminuição ou cessação do tabagismo para os fumantes ${ }^{17}$.

Evidências demonstram que os fatores ambientais modificáveis que interferem nas doenças cardiovasculares incluem alimentação inadequada, obesidade, sedentarismo e o hábito de fumar ${ }^{20}$. Os profissionais da saúde devem orientar as pessoas com doenças venosas a aderirem a tratamentos não farmacológicos em complemento aos farmacológicos para o sucesso da terapêutica. Dessa forma, é importante recomendar a mudança no estilo de vida, que inclui uma dieta adequada, a prática de exercício físico e a interrupção do hábito de fumar ${ }^{21}$.

A lesão neurológica em pessoas com DM, que envolve o sistema nervoso periférico nos seus componentes sensitivo-motor e autonômico, pode estar relacionada com as hipóteses clínicas de caráter metabólico e/ou microvascular ${ }^{22}$. Fatores preditivos para a ocorrência da neuropatia autonômica diabética são o controle glicêmico inadequado, presença de neuropatia diabética periférica, hipertensão arterial, obesidade, tabagismo e níveis de triglicerídeos e colesterol alterados ${ }^{23}$.

Nesta perspectiva, as atividades relacionadas a manutenção de exercícios físicos regulares, mediante prévia avalição médica e de acordo com a atual condições dos pés, alimentação saudável e a diminuição ou cessação do tabagismo para os fumantes ${ }^{17}$ também podem ter sido importantes para a melhora na sensibilidade, especificamente no ESN, após a 
intervenção "Ensino do Cuidado com os Pés". Além dessas atividades, o uso de calçados adequados, que acomodem os pés com conforto e a utilização gradual de calçados novos, com o uso inicial de apenas duas horas por dia, podem ter influenciado a diminuição das dores ou desconforto nas pernas, pois evidências comprovam que o tratamento das consequências da neuropatia inclui a educação em saúde, os cuidados com os pés e a utilização de sapatos confortáveis e adequados, de preferência com bico largo ${ }^{24}$.

Estudo descritivo realizado no Paraná em amostra constituída por 80 pessoas com DM, mostrou falta de conhecimento de medidas preventivas para possíveis complicações nos pés ${ }^{25}$. Neste estudo, mais de $80 \%$ dos voluntários afirmaram que nunca receberam orientações para o autocuidado dos pés e, na mesma proporção, nunca tiveram seus pés avaliados por um profissional de saúde.

O cuidado integral da pessoa com DM, incluimdo a avaliação dos pés devem ser contínuo, a fim de detectar precocemente o risco para desenvolver as graves morbidades crônicas, e consequentemente, as alterações nos pés, tais como úlceras ${ }^{17}$. $\mathrm{O}$ apoio educacional é fundamental para que as pessoas com DM sejam capazes de tomar decisões e adquirir habilidades para o autocuidado ${ }^{26}$.

No Sudão, foi desenvolvido um estudo quase experimental em que a intervenção educativa realizada de forma individual para as pessoas com DM, foi embasada no treinamento de habilidades necessárias com a doença. Este estudo apresentou influência do conhecimento nas variáveis sinais e sintomas da doença, sinais e sintomas de hipoglicemia e hiperglicemia, causas e sinais de complicações nos pés, cuidados com os pés e importância dos exercícios. Além de impactar no conhecimento, os voluntários apresentaram-se conscientizados para o desenvolvimento do autocuidado ${ }^{27}$.

Assim, o conhecimento se faz importante na aquisição de habilidades. A informação transmitida ao paciente sobre as atividades benéficas para sua saúde pode desencadear intenções e atitudes mais positivas em relação ao autocuidado ${ }^{28}$. Como ora apresentado, este estudo demostrou melhora nos escores de intenção de cuidar após a intervenção educativa, o que é positivo, pois, as intenções sempre antecedem as ações ${ }^{18,28}$.

Não foram encontrados estudos que utilizassem o questionário do comportamento planejado: cuidado com os pés, contudo, o instrumento QCP-CP é fundamentado no pressuposto que, quanto maior a intenção de iniciar um comportamento, maior a probabilidade da sua realização. Ainda, as intenções são indicadores do quanto uma pessoa está disposta a tentar, o que esboça o esforço para a execução de um determinado comportamento ${ }^{18,28}$. 
Não houve melhora da variável "temperatura tissular", após as intervenções educativas "Ensino do cuidado com os pés" o que pode ser consequência de problemas intrínsecos, como a desmielinização da bainha de mielina, provocada pela hiperglicemia sustentada ${ }^{29}$ que poderia necessitar de um tempo maior de acompanhamento para sofrer interferências.

Outra variável que se manteve após as intervenções foi a "pressão plantar", estudiosos admitem há necessidade da introdução de tecnologias, tais como calçados especiais concomitante com as orientações de medidas preventivas ${ }^{30}$, o que não foi disponibilizado no presente estudo.

As limitações do estudo estão relacionadas a falta de acompanhamento diário dos fatores que interferem no controle metabólico e na adesão das atividades de autocuidado com os pés. As intervenções fundamentadas em processo de educação são, por si só, complexas e sofrem influência de diferentes fatores que dificultam o experimento.

Vale ressaltar que a amostra deste estudo era homogênia, os grupos apresentavam o mesmo grau educacional e, ainda assim, foi possível observar resultados benéficos no GT, após a intervenção educativa, o que torna além de uma prática de baixo custo, viável para ser desenvolvida junto as pessoas com DM, independente do grau de instrução e conhecimento.

\section{Conclusão}

Os resultados do presente estudo sugerem que a intervenção educativa "Ensino do cuidado com os pés" é uma estratégia que pode influenciar os indicadores de comprometimento dos pés, como pele e anexos, circulação e sensibilidade e, ainda, no conhecimento e na intenção de cuidar dos pés de pessoas com DM tipo 2.

\section{Referências}

1 Armstrong DG, Boulton AJM, Bus SA. Diabetic Foot Ulcers and Their Recurrence. N Engl J Med. 2017;376(24):2367-75. https://doi.org/10.1056/NEJMra1615439

2 International Working Group on the Diabetic Foot (IWGDF). The 2015 Guidance on prevention and management of foot problems in diabetes: development of an evidence-based global consensus. Diabetes Metab Res Rev. 2016;32(suppl 1):S2-6.

https://doi.org/10.1002/dmrr.2694

3 Walsh JW, Hoffstad OJ, Sullivan MO, Margolis DJ. Association of diabetic foot ulcer and death in a population-based cohort from the United Kingdom. Diabet Med.

2016;33(11):1493-98. https://doi.org/10.1111/dme.13054 
4 Vieira CPB, Araújo TME. Prevalence and factors associated with chronic wounds in older adults in primary care. Rev da Escola de Enf. USP. 2018;52:1-8. https://doi.org/10.1590/S1980-220X2017051303415

5 Figueira ALG, Gomes-Villas Boas LC, Coelho ACM, Foss-Freitas MC, Pace AE. Educational interventions for knowledge on the disease, treatment adherence and control of diabetes mellitus. Rev Latino-Am Enf. 2017;25:1-8. https://doi.org/10.1590/15188345.1648.2863

6 Oliveira ARC, Araujo TL, Costa AGS, Morais HCC, Silva VM, Lopes MVO. Evaluation of patients with stroke monitored by home care programs. Rev Esc Enferm USP. 2013;47(5):1147-53. https://doi.org/10.1590/S0080-623420130000500019

7 Gomes LC, Coelho ACM, Gomides DS, Foss-Freitas MC, Foss MC, Pace AE. Contribution of family social support to the metabolic control of people with diabetes mellitus: A randomized controlled clinical trial. Applied Nurs Res. 2017;36:68-76.

https://doi.org/10.1016/j.apnr.2017.05.009

8 Herdman TH, Kamitsuru S. Diagnósticos de enfermagem da NANDA-I: definições e classificação 2018-2020. Porto Alegre: Artmed; 2018.

9 Johnson M, Moorhead S, Maas ML, Swanson E. NOC - Classificação dos Resultados de Enfermagem. 5 th ed. Amsterdã: Elsevier; 2016.

10 Bulechek GM, Butcher HK, Dochterman J, Wagner CM. Nic - Classificação Das Intervenções de Enfermagem. 6 th ed. Amsterdã: Elsevier; 2016.

11 Park H. Identifying Core NANDA-I Nursing Diagnoses, NIC Interventions, NOC Outcomes, and NNN Linkages for Heart Failure. Int J Nurs Knowl. 2014;25(1):30-8. https://doi.org/10.1111/2047-3095.12010

12 UK Prospective Diabetes Study (UKPDS) Group. Effect of intensive blood-glucose control with metformin on complications in overweight patients with type 2 diabetes. Lancet. 1998;352(9131):854-65. https://doi.org/10.1016/S0140-6736(98)07037-8

13 Folstein MF, Folstein SE, Mchugh PR. Mini-Mental State: a practical method for grading the cognitive state of patients for the clinician. J Psychiatr Res. 1975;12(3):189-98. https://doi.org/10.1016/0022-3956(75)90026-6

14 Medronho AR, Carvalho DM, Bloch KV, Luiz RR, Werneck GL. Epidemiologia. São Paulo: Atheneu; 2011.

15 Silva NCM, Chaves ECL, Carvalho EC, Iunes DH. Instrument for assessing tissue integrity of the feet of patients with diabetes mellitus. Acta Paul Enfer. 2013;26(6):535-41. https://doi.org/10.1590/S0103-21002013000600005

16 Monteiro LA, Souza VHS, Moreira JB, Pace AE, Iunes DH, Chaves ECL. Atividades para o "Ensino do autocuidado com os pés" de pessoas com Diabetes Mellitus. Rev Nursing. 2018: $1-17$. 
17 Brasil. Ministério da Saúde. Secretaria de Atenção à Saúde. Departamento de Atenção Básica. Manual do pé diabético. 1 st ed. Brasília: Ministério da saúde; 2016.

18 Monteiro LA, Chaves ECL, Souza VHS, Nogueira DA, Pereira MG, Iunes DH. Questionnaire of Planned Behavior in Diabetes - Foot Care: Validation. Online Braz J Nurs. 2015;14(3):255-62. https://doi.org/10.17665/1676-4285.20155159

19 Sharoni SKA, Rahman HA, Minhat HS, Ghazali SS, Azman MH. A self-efficacy education programme on foot self-care behaviour among older patients with diabetes in a public long-term care institution, Malaysia: a Quasi-experimental Pilot Study. BMJ Open 2017;7:e014393. https://doi.org/10.1136/bmjopen-2016-014393

20 Bernardo AFB, Rossi RC, Souza NM, Pastre CM, Vanderlei LCM. Association between physical activity and cardiovascular risk factors in individuals undergoing cardiac rehabilitation program. Rev Bras Med Esporte. 2013;19(4):231-5. https://doi.org/10.1590/S1517-86922013000400001

21 Kalanuria AA, Nyquist P, Ling G. The prevention and regression of atherosclerotic plaques: emerging treatments. Vasc Health Risk Manag. 2012;8(1):549-61. https://doi.org/10.2147/VHRM.S27764

22 Foss-Freitas MC, Junior WM, Foss MC. Autonomic neuropathy: a high risk complication for type 1 diabetes mellitus. Arq Bras Endocrinol, Metab. 2008;52(2):398-406. https://doi.org/10.1590/S0004-27302008000200028

23 Valensi P, Paries J, Attali JR. French Group for research and Study of Diabetic neuropathy. Cardiac Autonomic Neuropathy in Diabetic Patients: influence of diabetes duration, obesity and microangiopathic complications - The French Multicenter Study. Metabolism. 2003;52:815-20. https://doi.org/10.1016/s0026-0495(03)00095-7

24 Boulton AJ. The pathogenesis of diabetic foot problems: an overview. Diabet Med. 1996; 13:S12-6.

25 Carlesso GP, Gonçalves MHB, Júnior DM. Evaluation of diabetic patients' knowledge about preventive care of the diabetic foot, in Maringá, PR, Brazil. J Vasc Bras.

2017;16(2):113-118. https://doi.org/10.1590/1677-5449.006416

26 Contreras F, Sánchez M, Martínez MS, Chávez M, Mindiola A, Bermudez V, et al. Management and Education in Patients with Diabetes Mellitus. Medical \& Clinical Rev. 2017;3(2):1-7. https://doi.org/10.21767/2471-299X.1000049

27 MakkiAwouda FO, Elmukashfi TA, Al-Tom SAH. Effects of Health Education of Diabetic Patient's Knowledge at Diabetic Health Centers, Khartoum State, Sudan: 2007-2010. Glob J of Health Sci. 2014;6(2):221-226. https://doi.org/10.5539/gjhs.v6n2p221

28 Martins ECB, Serralvo FA, João BN. Teoria do comportamento planejado: uma aplicação no mercado educacional superior. Gestão e Regional. 2014;30(88):107-22.

https://doi.org/10.13037/gr.vol30n88.2292 
29 Boulton AJM, Armstrong DG, Albert SF, Frykberg RG, Hellman R, Kirkman MS et al. Comprehensive Foot Examination and Risk Assessment. Diabetes Care. 2008;31(8):1679-85. https://dx.doi.org/10.2337\%2Fdc08-9021

30 Oliveira AF, Marchi ACB, Leguisamo CP, Baldo GV, Wawginiak TA. Estimate cost on diabetic foot treatment, as prevent and save resources. Ciênc \& Saúde Col. 2014;19(6):166371. https://doi.org/10.1590/1413-81232014196.09912013 